\title{
Use of Bayesian population viability analysis to assess multiple management decisions in the recovery programme for the Endangered takahe Porphyrio hochstetteri
}

\author{
Danilo Hegg, Darryl I. MacKenzie and ian G. Jamieson
}

\begin{abstract}
Population modelling is an invaluable tool for identifying effective management strategies for threatened species whose populations are too small for experimental manipulation. Recently developed Bayesian approaches allow us to combine deterministic models with probability distributions to create stochastic models that account for uncertainty. We illustrate this approach in the case of the takahe Porphyrio hochstetteri, an Endangered flightless rail, which is supported by one of New Zealand's costliest recovery programmes. Using mark-recapture and logistic regression models implemented in a Bayesian framework we calculated demographic parameters for a fully stochastic population model based on 25 years of data collected from the last wild population of takahe in the Murchison Mountains, Fiordland. Our model results show that stoat trapping, captive rearing and cross-fostering of eggs/chicks in wild pairs all have a positive effect on takahe demography. If it were not for these management actions the Fiordland population would probably be declining $(\lambda=0.985$; confidence interval, $\mathrm{CI}=0.39-1.08$ ), with a non-negligible risk of quasi-extinction $(\mathrm{P}=16 \%)$ within 20 years. The captive rearing of eggs and chicks has been the main factor responsible for the positive growth observed during the last decade but in the future expanding stoat trapping to cover the entire Murchison Mountains would be the single most beneficial management action for the takahe population $(\lambda=1.038 ; \mathrm{CI}=0.86-1.10)$, followed by captive rearing $(\lambda=1.027$; CI $=0.85-1.09)$.
\end{abstract}

Keywords Captive rearing, mark-recapture, New Zealand, Porphyrio hochstetteri, predator control, rail, takahe

This paper contains supplementary material that can be found online at http://journals.cambridge.org

DANilo HeGg and IAN G. JAMIESON (Corresponding author) Department of Zoology, University of Otago, PO Box 56, Dunedin, New Zealand.

E-mail ian.jamieson@otago.ac.nz

Darryl I. MacKenZie Proteus Wildlife Research Consultants, Dunedin, New Zealand

Received 18 July 2011. Revision requested 8 November 2011.

Accepted 9 November 2011.

\section{Introduction}

R ecovery programmes for threatened species often R require implementing more than one management action to safeguard the species against certain extinction. It is important, however, to determine the effectiveness of each management action, in isolation or in combination with other actions, to assess past management strategies and to justify funding for current and future programmes.

Provided good demographic data are available, population viability analysis (PVA) can be a useful approach for identifying changes resulting from management decisions relative to baseline conditions (Beissinger \& Westphal, 1998). One problem associated with the use of PVA models is the necessity of dealing with uncertainty. Bayesian approaches combined with increased computational power provide a more rigorous method for dealing with uncertainty (e.g. Goodman, 2002; Dupuis \& Schwarz, 2007), allowing the calculation of probability distributions as opposed to mean values and confidence intervals for demographic parameters. Wade (2000) and Maunder (2004) illustrated the use of PVAs in a Bayesian framework on hypothetical datasets. Examples of their use in real-life scenarios to evaluate the effectiveness of long-term recovery programmes of threatened species include fisheries (e.g. Kinas, 2002; Pestes et al., 2008) or their by-catch (e.g. Zador et al., 2008), marsupials (e.g. Lindenmeyer \& McCarthy, 2006) and whooping cranes Grus americana (Moore et al., 2010). In this study we use a stochastic population model based on Bayesian parameter estimates to assist management decision-making for the threatened takahe Porphyrio hochstetteri. The takahe is a flightless rail categorized as Endangered on the IUCN Red List (BirdLife International, 2008) and as Nationally Critical in the New Zealand threat classification system (Miskelly et al., 2008). The takahe benefits from one of New Zealand's costliest recovery programmes (see below).

The takahe was widespread throughout New Zealand's South Island in pre-Polynesian times but its distribution became restricted to Fiordland as a consequence of hunting and habitat modification by early Polynesians (Trewick \& Worthy, 2001; Grueber \& Jamieson, 2011) and by the end of the 19th century it was considered extinct. The takahe was rediscovered in 1948 in the Murchison Mountains (Ballance, 2001); soon thereafter a $518 \mathrm{~km}^{2}$ Special Takahe Area was set 


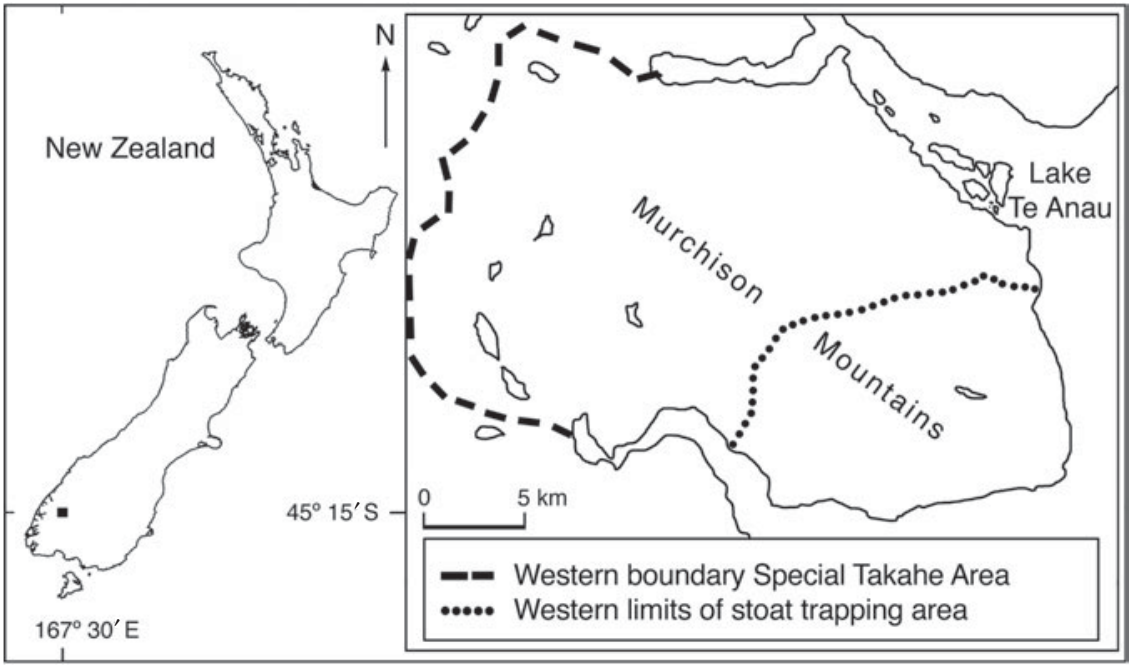

FIg. 1 Map of Murchison Mountains in Fiordland, New Zealand, showing boundary of Special Takahe Area and stoat control area. (Sourced from Topo250 Map 25 Te Anau, Land Information New Zealand. Crown Copyright Reserved). The black rectangle on the map of the South Island indicates the location of the main map. aside for its conservation (Fig. 1). Despite the relatively intact habitat the Murchison Mountains takahe population still faced some major challenges, including competition for food by introduced red deer Cervus elaphus (Mills et al., 1989), predation by introduced stoats Mustela erminea (Hegg et al., 2012), and the harsh climate in an alpine region at the periphery of the bird's original range (Maxwell \& Jamieson, 1997; Maxwell, 2001). Consequently, the takahe population appeared to continue to decline after its rediscovery and reached a low of 120 individuals in 1981 (Maxwell, 2001).

Management efforts initiated in the 1980 s to save the takahe from extinction included deer culling, predator trapping, nest manipulation, captive rearing for release into the wild and relocation of the birds to islands free of introduced pests and predators. An experimental stoat control programme, covering c. $40 \%$ of the Murchison Mountains (15,000 ha; Fig. 1), was conducted between 2002 and 2008, enabling a comparison of adult survival between trapped and trap-free areas (Hegg et al., 2012). Nest manipulation is limited to the removal of infertile or addled eggs from nests, to encourage re-nesting or to focus parental investment on the remaining viable eggs (Hegg et al., 2012). The artificial incubation of wild-sourced eggs and captive rearing of juveniles was originally designed to improve the low survival of eggs and chicks in the wild (Eason \& Willans, 2001) and assumes that second eggs from two-egg clutches are surplus eggs that can be removed from nests without lowering the productivity of the wild population.

Under this management regime the takahe population increased from 120 individuals in 1981-1982 to 297 individuals in 2006-2007, although about half of this growth was due to the creation of new populations on small offshore islands where further expansion of numbers is limited. The greatest potential for future expansion is still in the Fiordland population, which increased to 168 birds by 2006-2007 before dropping to 93 individuals in the
$2007 / 2008$ season as a consequence of an unprecedented stoat plague during the winter of 2007 (Hegg et al., 2012). Stoat plagues are associated with an increased supply of beech (Nothofagus spp.) mast that causes rodents, the stoat's main prey, to irrupt (King, 1983).

In a concurrent study we modelled takahe demographic data in the Murchison Mountains as a function of both environmental factors (e.g. beech seedfall, tussock seeding, winter temperature, rain and snowfall) and specific management activities (nest manipulation, captive rearing and stoat control; Hegg et al., 2012). These results are used here to build a stochastic population model to simulate population growth in Fiordland between 1982 and 2007 (i.e. up until the stoat plague), and to assess whether the observed modest increase in takahe numbers has been caused by effective management or by a combination of favourable climatic conditions. The same model is then used to project future population growth under different management scenarios, including extending stoat trapping from 40 to $95 \%$ of the Murchison Mountains, with the purpose of identifying the management strategy yielding maximum growth and minimizing the risk of extinction.

\section{Methods}

The survival and breeding success analyses were based on 16 years (1992-2007) and 25 years (1981-2005) of monitoring data, respectively. The takahe population in the Murchison Mountains was surveyed twice per year, in October/ November and in February/March, during which time banded adults were recorded and nests were managed (i.e. non-viable eggs removed and viable eggs fostered between pairs or transferred to the captive-rearing unit). Captivereared juveniles are released back into the wild at c. 1 year old. A detailed explanation of the field methods used to collect the data is reported elsewhere (Hegg et al., 2012). 


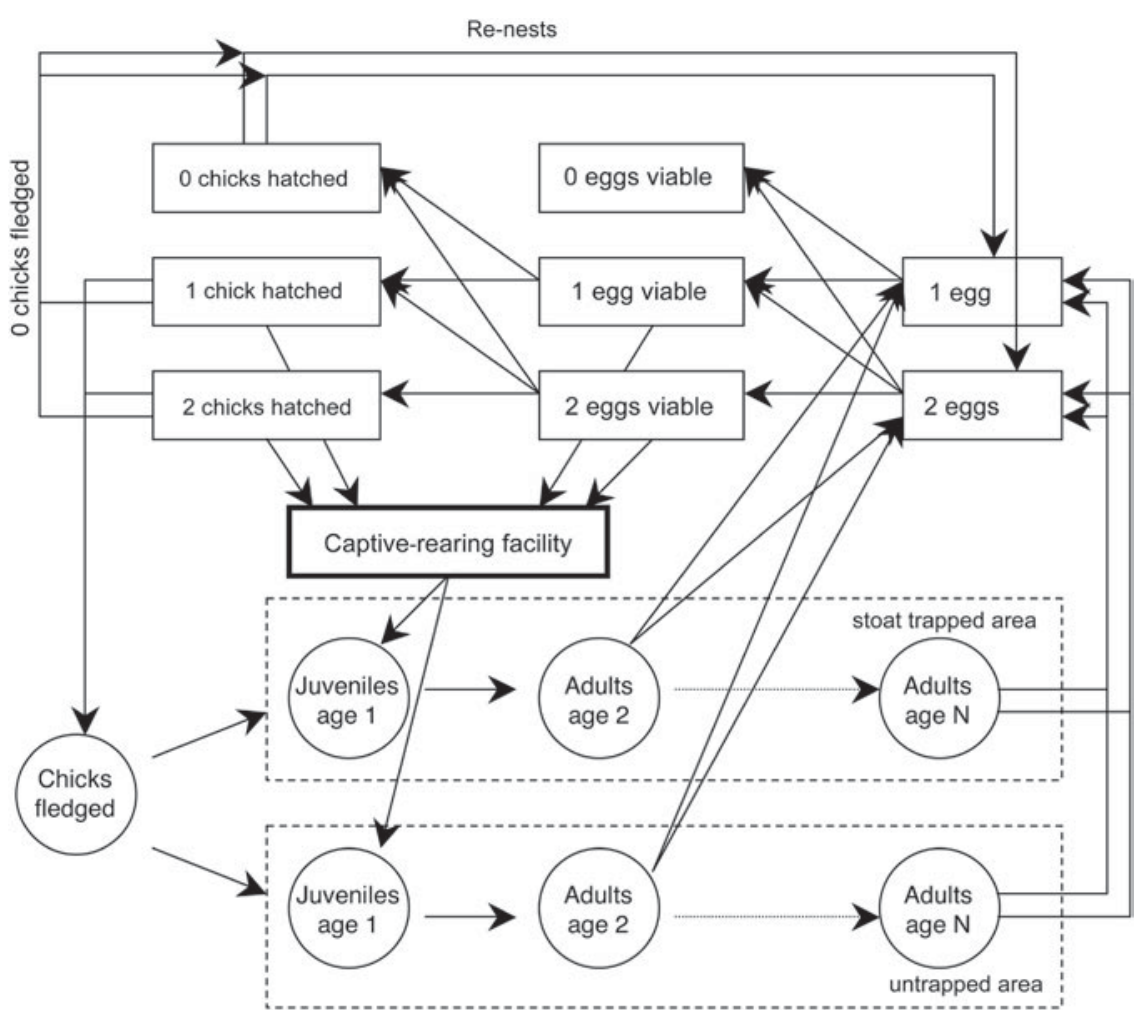

Fig. 2 Life-cycle graph for the takahe Porphyrio hochstetteri in the Murchison Mountains (Fig. 1), built into a Matlab population model. Transitions between circles are annual steps and all other transitions occur within a breeding season.
The population model developed for the takahe in the Murchison Mountains is a female-only, age structured birth-pulse model based on post-breeding census data (Caswell, 2001). The selection of a female-only model is based on the assumption that females drive the population dynamics and that there are always enough males available to fertilize all the females. The model assumes age-specific survival rates; reproductive output is also age dependent. A birth-pulse model assumes that the age distribution is a discontinuous series of pulses and that individuals reproduce on their birth day, a common model assumption for species with well-defined breeding seasons matching climatic factors (Caswell, 2001). Finally, a post-breeding census model takes a snapshot of the population every year just after reproduction, with the youngest age class composed of chicks that are not breeding in the current season. A life-cycle graph for the model is shown in Fig. 2.

The fully stochastic model includes parameter uncertainty and environmental and demographic stochasticity. Parameter uncertainty reflects two sources of sampling error in parameter estimates: firstly, we measure the outcome of a stochastic process and not the true probability of the underlying event and, secondly, we are rarely able to count all individuals or nests in a population (White et al., 2002). Environmental stochasticity accounts for the component of parameter variation over time that we are not able to explain and assume to be random, and demographic stochasticity accounts for an individual variation in fitness (Engen et al., 1998; Lande, 2002).
All demographic parameters $P$ were calculated as:

$$
g(P)=\alpha_{0}+\sum_{i} \alpha_{i} F_{i}+\varepsilon(t)
$$

where $g(P)$ is a transformation function to ensure demographic parameters are on the correct scale, $\alpha_{i}$ are the model parameters, $F_{i}$ are the factors included in the model (environment, management) and $\varepsilon(t)$ is a random time effect (environmental stochasticity), normally distributed with a mean of zero and unknown variance $\sigma^{2} \cdot \alpha_{i}$ and $\sigma^{2}$ were estimated in a Bayesian framework using WinBUGS 1.4.1 (Lunn et al., 2000) (100,00o iterations, 4,000 burn-ins). MATLAB v. R2006a (MathWorks, Natick, USA) was used to perform all population model simulations, drawing values at random from the posterior distributions generated in WinBUGS. Demographic stochasticity was simulated by applying all demographic parameters (survival, reproductive success) through a binomial operator. Each model scenario was simulated with 10,000 repetitions.

The values of the survival model parameters are summarized in Appendix, Table A.1, and the mean survival values are reported in Table A.2. Adult survival was modelled using the equation:

$$
\begin{aligned}
\operatorname{logit}(S)= & \alpha_{1}+\text { Adult } \cdot\left(\alpha_{2}+\alpha_{3} \text { Age }+\alpha_{4} \text { Age } e^{2}\right) \\
& +\alpha_{5} \text { Trapped }+\alpha_{6} \text { Seedfall }+\alpha_{7} \text { Tussock } \\
& +\alpha_{8} \text { Temperature }+\alpha_{9} \text { Rain }+\alpha_{10} \text { Snow }+\varepsilon(t)
\end{aligned}
$$

where $S$ is the survival rate from one year to the next; Adult takes a value of 1 if a bird is older than 1 year and is otherwise 
set to o; Age is a bird's age in years; Trapped takes a value of 1 for birds in the stoat control area, o for birds in the untrapped area; Seedfall is the annual beech seedfall measured as seeds per $\mathrm{m}^{2}$; Tussock is the percentage of flowering tussocks; Temperature is the mean temperature $\left({ }^{\circ} \mathrm{C}\right)$ in the three coldest months of the year; Rain is the total precipitation $(\mathrm{mm})$ in the calendar year; Snow is the total precipitation $(\mathrm{mm})$ from May to September, as a proxy for snowfall; all environmental variables were standardized. $\varepsilon(t)$ is a random error, normally distributed and with an average value of $o$ and unknown variance $\sigma^{2}$. A detailed explanation of the underlying methods and assumptions for the survival data is reported elsewhere (Hegg et al., 2012).

Most population models for birds look at breeding success as the combination of productivity to hatching (number of eggs hatched per pair) and fledging success (number of chicks fledged per egg hatched), or simply as the number of chicks fledged per year. A more detailed analysis of the breeding process was required for the purpose of this study because management of a nest depends on clutch size and egg viability (Hegg et al., 2012). A number of steps in the breeding process of the takahe were thus analysed separately as shown in Fig. 2. The mean values of takahe productivity used in the model are reported in Appendix, Table A.3. A detailed analysis of takahe breeding success is presented in Hegg et al. (2012).

The probability of a bird breeding as a function of age was modelled according to Equation 3, below, with the best fit model parameters being $\alpha_{\mathrm{O}}=2.00$ (confidence interval, CI, 1.73-2.27) and $\alpha_{1}=-4.32$ (CI $-5.08--3.60$; see also Appendix, Fig. A.1)

$$
\operatorname{logit}\left(P_{\text {Breeding, Age }}\right)=\alpha_{0}+\frac{\alpha_{1}}{\text { Age }}
$$

The above analysis was based on 23 years of data (19822004) for island takahe (C. Grueber \& I. Jamieson, unpubl. data), as data on breeding age of Fiordland takahe are too incomplete for a meaningful statistical analysis (Hegg, 2008). For model selection for different age-dependent models, see Appendix, Table A.4. The oldest breeding bird recorded in the Murchison Mountains was aged 13 years.

Within the range of historical population counts the effect of population density is not detectable for adult and juvenile takahe survival rates. There is some evidence that the probability of nesting, re-nesting and egg viability are negatively affected at high population numbers (Hegg et al., 2012), suggesting that population growth is limited by the number of available breeding territories. Therefore a spacelimited model for territorial species (Murray, 1979) was chosen to describe limited population growth for the takahe at high population density. This model assumes that survival rate and breeding success remain constant regardless of population density but the number of breeding pairs is capped and limited by the number of available territories, i.e. any excess adults will survive but will not breed. This model also yields a sigmoid population growth curve similar in shape to a logistic growth curve (Murray, 1979). For the purpose of this study, carrying capacity was defined as the maximum number of available breeding territories based on previous habitat surveys (D. Crouchley, unpubl. data).

The population model was coded in MATLAB. Software was developed that allowed the user to save and retrieve model scenarios, to display the values of all demographic parameters and plot them as a function of time and age, and to model and compare different management scenarios without having to alter the code. The number of model repetitions $\left(N_{\text {rep }}\right)$ can be specified by the user. For each repetition the parameters $\alpha_{i}$ and $\sigma^{2}$ in Equations 1-3 were randomly sampled from the posterior distributions generated in WinBUGS. To account for any correlation amongst factors within each logistic regression equation the joint posterior distributions were sampled, with all parameter values being from a single sampled row of the stored results rather than being randomly selected from independent distributions.

The observed values of the environmental factors were used to calculate demographic parameters when simulating historical trends in population growth. When simulating future population growth environmental factors were generated by bootstrapping of the environmental data collected over the last 25 years, with the additional constraint of heavy beech mast fall occurring on average every 4-5 years (Wardle, 1984) and tussock flowering events every 2-4 years (Mark, 1968). For this purpose all historical environmental data were classified into either non-mast year or mast year, and environmental factors sampled at random from either group, depending on whether it was a mast year.

A binomial operator was applied to calculate the probability of success (i.e. probability of hatching, fledging and survival), with the number of individuals being the number of trials. It was simpler to calculate the number of individuals $N_{j+1}$ in the year $j+1$ by summing and taking the difference $\left(N_{j+1}=N_{j}+\right.$ fledglings $_{j}$-deaths, with all addends being vectors of size $N_{\text {rep}}$ ) rather than using a Leslie matrix (Caswell, 2001). The population growth rate was then calculated as

$$
\lambda_{j}=N_{j+1} / N_{j}
$$

and the mean population growth rate as the geometric mean of the annual values $\lambda_{j}$ over the whole time interval simulated by the model.

In the results we report mean $\lambda$ (with 95\% creditability intervals) for each management scenario (see below), as well as the probability $(\mathrm{P})$ that $\lambda>1$ (i.e. positive growth). Furthermore, the mean growth rates $\lambda_{1}$ and $\lambda_{2}$ obtained under two different management scenarios were compared by running the model twice, once for each scenario, each 


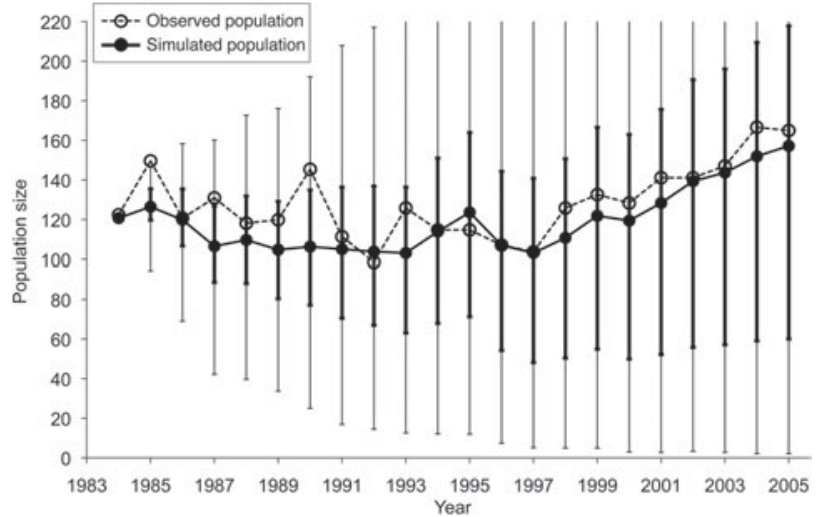

FIG. 3 Observed and simulated population trends of takahe, including captive rearing, in the Murchison Mountains (Fig. 1) from 1984 to 2005. Simulated results are median values with credibility intervals (from 10,000 repetitions), showing central 95\% (thin vertical bars) and 50\% (thick vertical bars) of the predicted distribution. Releases from the captive-rearing facility into the Murchison Mountains started in 1992.

time with the same number of repetitions and using the same strings of random numbers to select from the distributions of all model parameters. The difference $\Delta=\lambda_{1}-\lambda_{2}$ is also a vector of size $N_{\text {rep }}$, and the probability $\mathrm{P}\left(\lambda_{1}>\lambda_{2}\right)$ is the number of positive elements in $\Delta$ divided by $N_{\text {rep }}$. Finally, we also report the probability of quasiextinction (Ginzburg et al., 1982), which we defined as the population declining to 20 individuals or less by the end of the simulation period.

The model was run for two different time periods. (1) Retrospectively, for the last 25 years, from an observed starting point of 120 individuals. Survival rates and reproductive success were calculated as a function of the observed environmental factors (Hegg et al., 2012). The model results were then matched with the actual population counts, which include unbanded and unidentified birds not included in the demographic analyses. (2) Into the future, to project population growth in the next 20 years. The future environmental factors were simulated, through bootstrapping of the observed data.

Different management scenarios were modelled based on different levels (o, 40 or 95\%) of stoat trapping in the Special Takahe Area and captive-rearing effort (frequency of operation every year, and every second or third year, and no captive rearing). The 2008 management scenario (40\% of Murchison Mountains trapped, captive rearing every year) was used as a reference scenario for comparison with other management options, as 2008 is when this analysis was completed. A hypothetical fostering scenario was also modelled, in which any nests with two viable eggs have one egg removed and transferred to a nest that only produced non-viable eggs. This is expected to improve the reproductive output, as two-egg clutches have a lower hatching and fledging success than one-egg clutches
(Hegg et al., 2012). When simulating the effects of captive rearing and release the proportion of nests in which one and two eggs or chicks were removed was based on an analysis of historical egg/chick transfers to the captive-rearing facility in Burwood (Hegg, 2008). The maximum number of eggs or chicks transferred into captivity was also limited to 20 , the maximum capacity of the captive-rearing facility.

\section{Results}

\section{Simulation of historical population trends}

The simulated results of historical population trends compare well with the observed data (Fig. 3). The model suggests a population that is steady or slowly declining throughout the 1980 s and early 1990s, with a positive population growth during the last decade. The observed decline from 1993 to 1997 coincides with a series of particularly cold winters.

A hypothetical scenario without captive rearing is shown for comparison in Fig. 4; it suggests that captive rearing suppressed population growth prior to 1992 . This is because eggs were being transferred into captivity but no captivereared chicks were released into the Murchison Mountains until 1992. Instead, chicks were initially released into the Stewart Mountains in an attempt to start a new population (Maxwell, 2001). The model also indicates that management has had a significant positive effect during the last decade, with the actual population size being $22 \%$ higher than it would have been without the captive-rearing programme (Fig. 4). The probability that the current population size is larger than it would have been without the captive-rearing programme is $\mathrm{P}=98.4 \%$ (10,000 model repetitions). There is an $85.7 \%$ probability that the gain generated by the captive-rearing programme is at least $10 \%$, and a probability of $75.4 \%$ that the gain is at least $20 \%$. Under the current management scenario there was an $84.4 \%$ probability of a positive population growth rate since 1992 (the start of releases of captive-reared birds into the Murchison Mountains). The probability of positive growth rate in the absence of management would have been only $66.5 \%$.

\section{Growth projection and evaluation of current management strategies}

The projected population growth rates with and without stoat trapping and captive rearing are reported in Table 1, scenarios $\mathrm{A}$ to $\mathrm{D}$. A value of $\lambda<_{1}$ (declining population) is expected in the absence of captive rearing and stoat trapping (D). The population growth is likely to be positive with the aid of the captive-rearing programme (B), and is significantly improved by the stoat trapping programme (A). Stoat trapping alone at the 2008 level (covering $40 \%$ of 
TABLE 1 Predicted growth rates $(\lambda)$ and probability of quasi-extinction for the Murchison Mountains takahe population 20 years into the future, under different management scenarios. 'Quasi-extinction' is defined as the population size dropping to $<20$ individuals.

\begin{tabular}{|c|c|c|c|}
\hline Scenario & $\lambda$ & $\begin{array}{l}\mathrm{P} \lambda>1 \\
(\%)\end{array}$ & $\begin{array}{l}\text { P Quasi-extinction } \\
(\%)\end{array}$ \\
\hline $\begin{array}{l}\text { A. } 2008 \text { management scenario (deer control + captive rearing + stoat trapping } 40 \% \text { of } \\
\text { area) }\end{array}$ & $1.043(0.88-1.10)$ & 78.3 & 3.3 \\
\hline B. Deer control + captive rearing & $1.027(0.85-1.09)$ & 66.6 & 5.7 \\
\hline C. Deer control + stoat trapping ( $40 \%$ of area) & $1.005(0.79-1.09)$ & 52.4 & 10.0 \\
\hline D. Deer control only & $0.985(0.39-1.08)$ & 41.9 & 15.9 \\
\hline E. Deer control + captive rearing + stoat trapping $95 \%$ of area & $1.062(0.92-1.11)$ & 89.0 & 1.2 \\
\hline F. Deer control + stoat trapping $95 \%$ of area & $1.038(0.86-1.10)$ & 73.8 & 4.6 \\
\hline G. Deer control + fostering + stoat trapping $95 \%$ of area & $1.049(0.86-1.11)$ & 75.9 & 4.0 \\
\hline H. Deer control + fostering + stoat trapping $40 \%$ of area & $1.022(0.82-1.10)$ & 62.2 & 7.7 \\
\hline $\begin{array}{l}\text { I. Deer control + captive rearing every second year, max. } 20 \text { eggs/chicks + stoat } \\
\text { trapping } 40 \% \text { of area }\end{array}$ & $1.026(0.86-1.09)$ & 65.5 & 4.7 \\
\hline $\begin{array}{l}\text { J. Deer control + captive rearing every third year, max. } 20 \text { eggs/chicks + stoat trapping } \\
40 \% \text { of area }\end{array}$ & $1.022(0.84-1.09)$ & 61.5 & 6.5 \\
\hline $\begin{array}{l}\text { K. Deer control + captive rearing every year, max. } 30 \text { eggs/chicks + stoat trapping } 40 \% \\
\text { of area }\end{array}$ & $1.049(0.90-1.10)$ & 81.2 & 2.3 \\
\hline
\end{tabular}

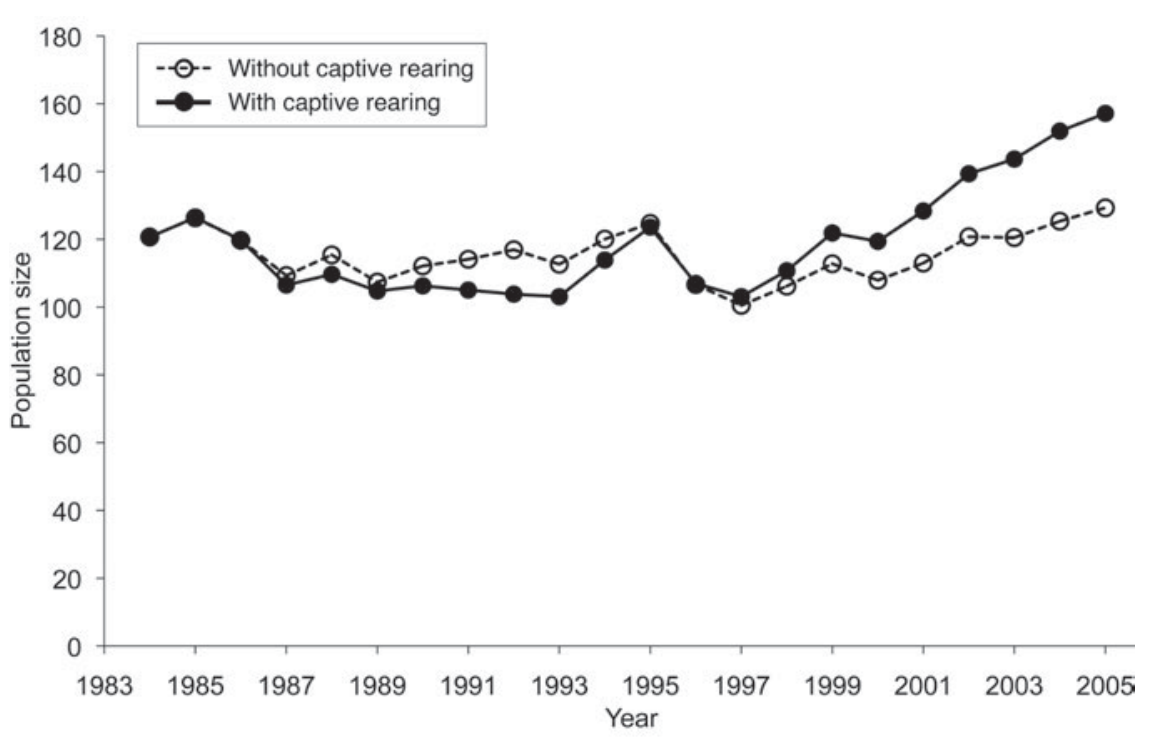

FIG. 4 Simulated population trend of takahe with and without captive rearing for the Murchison Mountains (Fig. 1) from 1984 to 2005. Simulated results show median values (with credibility intervals removed for clarity). Eggs and chicks were transferred from the Murchison Mountains to the captiverearing unit throughout the period but juveniles were released into the Stuart Mountains from 1987 to 1991 only. the Murchison Mountains) only gives 52\% probability of positive population growth $(\mathrm{C})$. The probability that the population growth rate would be lower than it is under the 2008 management scenario is $\mathrm{P}\left(\lambda_{\mathrm{B}}<\lambda_{\mathrm{A}}\right)=95.8 \%$ in the absence of stoat trapping, $\mathrm{P}\left(\lambda_{\mathrm{C}}<\lambda_{\mathrm{A}}\right)=99.0 \%$ in the absence of the captive-rearing programme, and $\mathrm{P}\left(\lambda_{\mathrm{D}}<\lambda_{\mathrm{A}}\right)=99.7 \%$ in the absence of both (Fig. 5 ).

\section{Extension of the stoat trapping programme}

The results indicate that the population growth rate would increase and the risk of extinction decrease with a larger trapped area (Table 1, scenarios E and F). A management scenario including both captive rearing and an expanded trapping programme (E) is the scenario with the highest probability of a positive population growth rate $\left(\mathrm{P} \lambda>_{1}=89 \%\right.$ ). If trapping covering $95 \%$ of the Murchison Mountains (F) and captive rearing (B) were mutually exclusive, then the trapping option would achieve a slightly larger population growth rate $\left(\lambda_{\mathrm{F}}=1.038\right.$; $\left.\mathrm{P} \quad \lambda_{\mathrm{F}}>_{1}=74 \%\right) \quad$ vs $\quad\left(\lambda_{\mathrm{B}}=1.027 ; \quad \mathrm{P} \quad \lambda_{\mathrm{B}}>_{1}=67 \%\right)$. The difference, however, is small and the level of confidence is moderate, as the probability that $\lambda$ is greater under the trapping rather than the captive-rearing scenario is $\mathrm{P}\left(\lambda_{\mathrm{F}}>\lambda_{\mathrm{B}}\right)=74 \%$.

\section{Fostering as an alternative to captive rearing?}

The fostering scenarios assumed that any nest with two viable eggs has one egg removed and transferred to a pair with infertile eggs (provided enough nests have been found that contain no viable eggs). The results (Table 1) suggest 


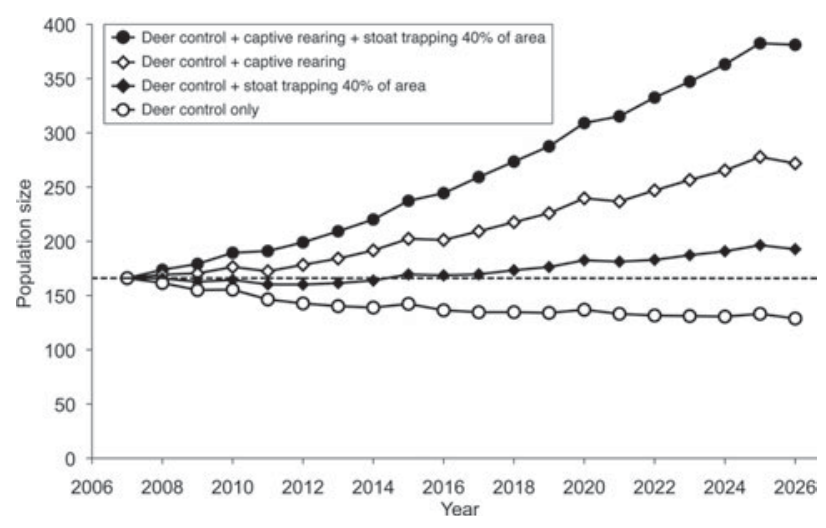

FIG. 5. Simulated population trends of takahe in the Murchison Mountains under four different management scenarios over a 20 -year period. Results are median values based on 10,000 model repetitions (with credibility intervals removed for clarity). Deer and stoat control and captive rearing represent the 2008 management scenario.

that fostering $(\mathrm{H})$ would be beneficial compared to a scenario of no nest management (C) $\left(\lambda_{\mathrm{H}}=1.022\right.$, $\left.\lambda_{\mathrm{C}}=1.005 ; \mathrm{P} \lambda_{\mathrm{H}}>\lambda_{\mathrm{C}}=79.9 \%\right)$, but not as effective as the current captive rearing programme (A) $\left(\lambda_{\mathrm{A}}=1.043\right.$, $\left.\lambda_{\mathrm{H}}=1.022 ; \quad \mathrm{P} \quad \lambda_{\mathrm{A}}>\lambda_{\mathrm{H}}=77.3 \%\right)$. The probability that fostering in conjunction with an expanded stoat trapping programme $(\mathrm{G})$ results in a higher growth rate compared to the current 2008 management regime $\left(\lambda_{\mathrm{G}}>\lambda_{\mathrm{A}}\right)$ is $68.8 \%$.

\section{Alternative captive-rearing management options}

The operation of the captive-rearing facility in Burwood requires substantial funds, c. $20 \%$ of the total sum allocated for takahe management (K.A. Edge, pers. comm. 2006). Questions have been raised about whether it would be possible to reduce expenses by running the captive rearing programme every second or third year (assuming that this is logistically possible), and whether it would be beneficial to increase the capacity of the captive rearing facility from the current 20 eggs/chicks per season to 30 eggs/chicks per season.

Model simulations indicate that the benefits of a captiverearing programme diminish if it is not run continuously (Table 1, scenarios I and J). Running the programme every second year halves the gain compared to a yearly operation $\left(\lambda_{\mathrm{A}}=1.043, \quad \lambda_{\mathrm{I}}=1.026, \quad \lambda_{\mathrm{C}}=1.005 ; \quad \mathrm{P} \quad \lambda_{\mathrm{A}}>\lambda_{\mathrm{I}}=97.3 \%\right.$; $\mathrm{P} \lambda_{\mathrm{I}}>\lambda_{\mathrm{C}}=97.2 \%$ ), and running the programme every third year reduces the population growth rate by a further $20 \%\left(\lambda_{\mathrm{J}}=1.022, \lambda_{\mathrm{C}}=1.005, \mathrm{P} \lambda_{\mathrm{J}}>\lambda_{\mathrm{C}}=96.9 \%\right)$. Increasing the capacity of Burwood to 30 eggs/chicks per season (scenario $\mathrm{K}$ ) would make little difference $\left(\lambda_{\mathrm{K}}=1.049\right.$, $\left.\lambda_{\mathrm{A}}=1.043 ; \mathrm{P} \lambda_{\mathrm{K}}>\lambda_{\mathrm{A}}=89.0 \%\right)$, mainly because of the current limit on the supply of wild eggs/chicks.

In almost all cases a higher population growth rate corresponds to a lower risk of quasi-extinction (Table 1). Where this is not the case (e.g. scenarios A and G), the higher probability of quasi-extinction is associated with a higher level of uncertainty (i.e. wider credibility intervals).

\section{Discussion}

Before we interpret the results of the model in terms of past population trends and future growth, it is important to be explicit about the general limitations of PVA (Beissinger, 2002). The predicted population trends show extremely wide credibility intervals, which become wider the further they are projected into the future. This means that the model should not be used as a crystal ball: we are simply not able to predict confidently how many takahe will be alive in the Murchison Mountains in 20 years time. Although a slight increase in the population size is most likely under most management scenarios, extinction or a doubling of the population are not impossible (although highly unlikely). In contrast, we have a high level of confidence that the population growth will be greater under one management regime relative to another. The lack of accuracy of population models for absolute predictions and their reliability for relative predictions (i.e. comparisons of alternative scenarios) has been discussed in detail elsewhere (e.g. Beissinger \& Westphal, 1998; Coulson et al., 2001; Brook et al., 2002). Another limitation of the model lies in the fact that the probabilities of survival and breeding success are calculated as linear functions of population density and environmental factors and are valid only within the range for which they were calculated. This is especially true for the estimates of the probability of nesting and re-nesting and of egg viability, all of which are density dependent and were only calculated in a historical range between 100 and 170 individuals. However, this only affects the absolute population size predictions, not the comparisons between different scenarios. Similarly, a misjudgment of the carrying capacity would affect the population trajectories at population numbers above $\mathrm{K}$ but it would have no impact on the qualitative difference between alternative management scenarios.

With the above limitations in mind, the model gives valuable insights into the causes of historical population trends and the impact of different management scenarios on the future of the takahe. The simulated population trends in the Murchison Mountains over the last 2 decades (Fig. 4) indicated that population growth was suppressed prior to 1992 when the juveniles raised from eggs transferred into captivity were subsequently released into the nearby Stewart Mountains in a failed attempt to start a new population (Maxwell, 2001). Furthermore, the observed and simulated data (based on models including climatic factors) both show a significant drop in bird numbers in the mid 1990s, supporting the hypothesis that the population decline was because of extremely cold winters (Maxwell \& Jamieson, 1997; Maxwell, 2001). 
A reversal in trends with a steady population growth has followed since 1997. This increase in numbers during the last decade is in agreement with the model's predictions and suggests that the positive growth is largely because of the captive-rearing programme, with the actual population size being $22 \%$ higher than it would have been if there had been no captive rearing.

Simulated population trends under different management scenarios indicate that captive rearing of chicks in Burwood, fostering of one egg from nests with two viable eggs and stoat trapping are all significant improvements over the option of no management, the probability of positive population growth in the absence of management being $<50 \%$. Management is required, or the takahe population in Fiordland is highly likely to go extinct before the end of the century.

Although all of the above management actions are likely to improve the population growth rate to a value $>1$, stoat trapping and captive rearing of chicks seem to be the most effective actions, with fostering being only of moderate benefit. The best among the simulated scenarios assumes the Burwood captive-rearing facility operating at current capacity in conjunction with a stoat trapping programme covering 95\% of the Murchison Mountains. Under this management scenario there is a $90 \%$ probability of having a positive population growth rate. If we had to select only one management activity (because of limited funding and resources), stoat trapping over the entire Murchison Mountains would be more effective than the captive-rearing programme, and the captive-rearing programme would be more effective than the fostering of eggs. Stoat trapping ranks highest because it increases adult survival whereas the latter two strategies only affect the takahe's reproductive output. For a long-lived species with a low reproductive output such as the takahe, adult survival is always the most important factor in the population growth rate. Captive rearing of chicks is more effective than fostering because fledging success and survival over the first winter are higher in captivity than they are in the wild. Moreover, our models do not include potentially important factors such as parental quality. It may be that pairs that fail to lay eggs, or fail to complete incubation, are poorer parents, in which case the benefit of a fostering scenario would be less than predicted by our models.

Model results also indicate that, under the current level of stoat trapping ( $40 \%$ of the area), discontinuing captive rearing would result in a high risk of a population decline. In the long term, if the threat posed by stoats were effectively removed (i.e. the stoat trapping area was expanded to cover the entire Murchison Mountains), then the fostering of excess eggs to wild pairs might be a cost-effective complementary management option that could eventually replace the captive-rearing programme. There appears to be little benefit at present in increasing the capacity of the captive rearing unit to $>20$ eggs/chicks per season because the supply of takahe eggs is limited in the Murchison Mountains. Operating the facility every second year would save costs but any financial savings would be outweighed by the predicted lower population growth rate and by the logistical difficulties of retaining staff and expertise.

\section{Conclusions and recommendations}

Although taking up a significant proportion of the annual management budget for takahe there is little doubt that captive rearing has had a significant effect on the positive population growth observed over the last decade. We recommend that its operation should continue until important threats to the takahe in its natural environment are removed or are effectively controlled. Although an intermittent operation of the captive-rearing unit would make funds available for other activities (e.g. stoat trapping), depreciation and maintenance costs remain, and retaining trained staff and expertise would become problematic.

In spite of a stoat irruption during the winter of 2007 when available trap capacity in the Murchison Mountains was overwhelmed, we are confident that stoat trapping in most typical years is having a positive effect on adult survival of takahe as well as other vulnerable native species. If a recent extension of the trapping regime from 15,000 to 50,000 ha significantly reduces stoat densities and chances of periodic irruptions, it may eventually be possible to scale down the captive-rearing programme, and the fostering of surplus eggs could be trialled as a cost-effective alternative to captive rearing.

\section{Acknowledgements}

This project would not have been possible without the assistance of Jane Maxwell and Glen Greaves, who provided expertise on the takahe and its management and supplied the data used to calculate the demographic parameters. We thank Dave Crouchley, Kerri-Anne Edge, Phil Tisch, Nik Joice and Andrew Gormley, and a host of unnamed field workers over the last 3 decades, for their assistance. Henrik Moller and Richard Duncan provided helpful comments. This project was funded by the Takahe Recovery Programme's corporate sponsor Mitre10, the University of Otago and an Otago University postgraduate scholarship to $\mathrm{DH}$.

\section{References}

Ballance, A. (2001) Takahe: the bird that twice came back from the grave. In The Takahe-Fifty Years of Conservation (eds I.G. Jamieson \& W.G. Lee), pp. 18-22. University of Otago Press, Dunedin, New Zealand. 
Beissinger, S.R. (2002) Population viability analysis: past, present, future. In Population Viability Analysis (eds S.R. Beissinger \& D.R. McCullough), pp. 5-17. University of Chicago Press, Chicago, USA.

BeIsSingeR, S.R. \& West PHAL, M.I. (1998) On the use of demographic models of population viability in endangered species management. Journal of Wildlife Management, 62, 821-841.

Birdifine International (2008) Porphyrio hochstetteri. In IUCN Red List of Threatened Species v. 2011.1. Http://www.iucnredlist.org [accessed 8 July 2011].

Brook, B.W., Burgman, M.A., Akçakaya, H.R., O'Grady, J.J. \& FrankHaM, R. (2002) Critiques of PVA ask the wrong questions: throwing the heuristic baby out with the numerical bath water. Conservation Biology, 16, 262-263.

Caswell, H. (2001) Matrix Population Models: Construction, Analysis and Interpretation. Sinauer Associates, Massachusetts, USA.

Coulson, T., Mace, G.M., Hudson, E. \& Possingham, H. (2001) The use and abuse of population viability analysis. Trends in Ecology \& Evolution, 16, 219-221.

Dupuis, J.A. \& Schwarz, C.J. (2007) A Bayesian approach to the multistate Jolly-Seber capture-recapture model. Biometrika, 63, 1015-1022.

Eason, D.K. \& Willans, M. (2001) Captive rearing: a management tool for the recovery of endangered takahe. In The Takahe-Fifty Years of Conservation (eds I.G. Jamieson \& W.G. Lee), pp. 80-95. University of Otago Press, Dunedin, New Zealand.

Engen, S., BAKкe, Ø. \& Islam, A. (1998) Demographic and environmental stochasticity-concepts and definitions. Biometrika $54,840-846$.

Ginzburg, L.R., Slobodkin, L.B., Johnson, K. \& Bindman, A.G. (1982) Quasiextinction probabilities as a measure of impact on population growth. Risk Analysis, 21, 181-191.

Goodman, D. (2002) Predictive Bayesian population viability analysis: a logic for listing criteria, delisting criteria, and recovery plans. In Population Viability Analysis (eds S.R. Beissinger \& D.R. McCullough), pp. 50-85. University of Chicago Press, Chicago, USA.

Grueber, C. \& Jamieson, I.G. (2011) Low genetic diversity and small population size of Takahe Porphyrio hochstetteri on European arrival in New Zealand. Ibis, 153, 384-394.

HEGG, D. (2008) Use of a stochastic population model as a tool to assist decision making in the management of takahe (Porphyrio hochstetteri). MSc thesis. University of Otago, Dunedin, New Zealand.

Hegg, D., Greaves, G., Maxwell, J.M., MacKenzie, D.I. \& Jamieson, I.G. (2012) Demography of takahe (Porphyrio hochstetteri) in Fiordland: environmental factors and management affect survival and breeding success. New Zealand Journal of Ecology, 36, 75-89.

Kinas, P.G. (2002) The impact of incidental kills by gill nets on the Franciscana dolphin (Pontoporia blainvillei) in southern Brazil. Bulletin of Marine Science, 70, 409-421.

KING, C.M. (1983) The relationship between beech (Nothofagus spp.) seedfall and population of mice (Mus musculus), and the demographic and dietary responses of stoats (Mustela erminea), in three New Zealand forests. Journal of Animal Ecology, 52, 141-166.

LANDE, R. (2002) Incorporating stochasticity in population viability analysis. In Population Viability Analysis (eds S.R. Beissinger \& D.R. McCullough), pp. 18-40. University of Chicago Press, Chicago, USA.

Lindenmeyer, D.B. \& McCarthy, M.A. (2006) Evaluation of PVA models of arboreal marsupials: coupling models with long-term monitoring data. Biodiversity and Conservation, 15, 4079-4096.

Lunn, D.J., Thomas, A., Best, N. \& Spiegelhalter, D. (2000) WinBUGS-a Bayesian modelling framework: concepts, structure, and extensibility. Statistics and Computing, 10, 325-337.
MARK, A.F. (1968) Factors controlling irregular flowering in four alpine species of Chionochloa. Proceedings of the New Zealand Ecological Society, 15, 55-60.

Maunder, M.N. (2004) Population viability analysis based on combining Bayesian, integrated, and hierarchical analyses. Acta Oecologica, 26, 85-94.

Maxwell, J.M. (2001) Fiordland takahe: population trends, dynamics and problems. In The Takahe-Fifty Years of Conservation (eds I.G. Jamieson \& W.G. Lee), pp. 61-79. University of Otago Press, Dunedin, New Zealand.

Maxwell, J.M. \& Jamieson, I.G. (1997) Survival and recruitment of captive-reared and wild-reared takahe in Fiordland, New Zealand. Conservation Biology, 11, 683-691.

Mills, J.A., Lee, W.G. \& Lavers, R.B. (1989) Experimental investigations of the effects of takahe and deer grazing on Chionochloa pallens grassland, Fiordland, New Zealand. Journal of Applied Ecology, 26, 397-417.

Miskelly, C.M., Dowding, J.E., Elliott, G.P., Hitchmough, R.A., Powlesland, R.G., Robertson, H.A. et al. (2008) Conservation Status of New Zealand Birds, 2008. Notornis, 55, 117-135.

Moore, C.T., Converse, S.J., Folk, M.J., Runge, M.C. \& Nesbitt, S. A. (2010) Evaluating release alternatives for a long-lived bird species under uncertainty about long-term demographic rates. Journal of Ornithology, 152 (Suppl 2), S561-S572.

Murray, B.G. (1979) Population Dynamics-Alternative Models. Academic Press, New York, USA.

Pestes, L.R., Peterman, R.M., Bradford, M.J. \& Wood, C.C. (2008) Bayesian decision analysis for evaluating management options to promote recovery of a depleted salmon population. Conservation Biology, 22, 351-361.

Trewick, S.A. \& Worthy, T.H. (2001) Origins and prehistoric ecology of takahe based on morphometric, molecular, and fossil data. In The Takahe-Fifty Years of Conservation (eds I.G. Jamieson \& W.G. Lee), pp. 31-48. University of Otago Press, Dunedin, New Zealand.

WADE, P.R. (2000) Bayesian methods in conservation biology. Conservation Biology, 4, 1308-1316.

WARdLe, J.A. (1984) The New Zealand Beeches: Ecology, Utilisation and Management. New Zealand Forest Service, Wellington, New Zealand.

White, G.C., Franklin, A.B. \& Shenk, T.M. (2002) Estimating parameters of PVA models from data on marked animals. In Population Viability Analysis (eds S.R. Beissinger \& D.R. McCullough), pp. 169-19o. University of Chicago Press, Chicago, USA.

Zador, S.G., Punt, A.E. \& Parrish, J.K. (2008) Population impacts of endangered short-tailed albatross bycatch in the Alaskan trawl fishery. Biological Conservation, 141, 872-882.

\section{Appendix}

The appendix for this article is available online at http:// journals.cambridge.org

\section{Biographical sketches}

DANILO HEGG has worked for the takahe recovery programme and undertook this study as part of his MSc in Wildlife Management at Otago University. DARRYL MACKENZIE is a biometrician; his main area of interest in the use of occupancy models for monitoring and research. IAN JAMIESON is a conservation biologist with interest in reintroductions of threatened species. He is a long-serving member of the Takahe Recovery Group. 